\title{
Assessing the Blockage of Toxicity of Stereolithographically 3D-Printed Parts Coated with Parylene C
}

\section{Kaiyuan Huang ( $\square$ kyhuang@cau.edu.cn )}

College of Biological Science, China Agricultural University, Beijing, China

\section{Research Article}

Keywords: 3D printing, stereolithography, SLA, toxicity, zebrafish, acute toxicity test, reduce, block

Posted Date: August 30th, 2021

DOI: https://doi.org/10.21203/rs.3.rs-847107/v1

License: (c) (i) This work is licensed under a Creative Commons Attribution 4.0 International License.

Read Full License 


\section{Abstract}

Comparing to fused deposition modeling (FDM), the stereolithography (SLA) 3D printing method can provide higher fabrication speed and much better resolution, which is demanded by biomedical usage. However, the additives migrating from SLA printed plastic are toxic to creatures, and the method to reduce the toxicity by soaking and exposure to ultraviolet is inadequate, while current biocompatible photopolymerizing resins are rare and expensive. Here, we provide a method to nearly block all toxicity by coating the fabrication with parylene $\mathrm{C}$. We use early life zebrafish to assess the toxicity of the SLA printed petri dishes whether or not coated with parylene $C$. The result shows nearly no influence of the coated petri dishes cultured early life zebrafish development compared to control groups, while the fertilized embryos of uncoated groups get killed in 24 hours. The method provides a cheap and easy way to produce customized biocompatible fabrication, which contributes to the popularization of 3D printed products, either in daily life or in research.

\section{Introduction}

3D printing, or additive manufacturing, born in the mid-1990s, gains its widespread application in recent years [1]. Metal powder, ceram, beton, or photosensitive resin can be used as a consumable material for $3 \mathrm{D}$ printing. Through the patterning designed in advance on a computer, 3D printing can fabricate or accumulate consecutive layers of the consumable material and form a 3D entity eventually [2]. After decades years of development, there are several kinds of 3D printing methods, including fused deposition modeling (FDM), stereolithography (SLA), powder-liquid 3D printing (PLP), and selective laser sintering (SLS) [1-3]. FDM method provides many kinds of polymers with biocompatibility [4]. However, such biocompatibility is negated when the 3D parts are with increasing complexity [5]. Particularly, SLA has its advantages of extraordinary resolution, fabrication speed, and smooth finished surface $[3,6]$. Therefore, SLA is more widely applied in medical science and biology, such as tissue engineering, organ printing, artificial bones development, and drug delivery [3]. However, for SLA, only a tiny fraction of commercially available photo-polymerizing resins are certified as biocompatible (i.e., according to ISO 10993-5:2009 or USP class testing) at present. In addition, among the imperative compounds or additives for SLA, more than 20 compounds are proved to be poisonous for creatures and be found to remain in the finished product [7]. Some of the toxic compounds are known as photoinitiators. For example, 1-HCHPK leak from the 3D parts can cause developmental defects in zebrafish such as spine curvature, heart oedema, and an overt decrease in skin pigmentation [8]. Hence, people turn to attempt to reduce the toxicity of SLA 3D parts by treating with supercritical carbon dioxide [9], sonication of the material in isopropanol [10], and exposing to ultraviolet light [11]. However, the methods mentioned above are either complex, expensive, or inadequate.

To eliminate the influence of the toxicity of SLA 3D parts, we exploited a method of coating the 3D parts with parylene $\mathrm{C}$ to isolate the toxicity. Parylene is a generic name for the members of a xylylene family of polymers $[12,13]$. They are classified as thermoplastic polymers formed on substrate surfaces using a technique of vacuum deposition polymerization $[12,14]$. Currently, three of them are commercially 
valuable, i.e., parylene $\mathrm{N}$ and its halogenated derivatives and parylene $\mathrm{C}$ ( $\mathrm{p}$-xylylene substituted with a single chlorine molecule) and D (substituted with two chlorine molecules) [12, 14]. Parylene $C$ exhibits an excellent properties for medical applications, such as low water absorption, high dielectric constant, low coefficient of friction, and moderate elongation at break [12]. Until now, it has been successfully used as a protective coating on medical metals and metal alloys, such as gold, platinum [15-17], medical-grade stainless steel (SS 316L) [18-20], and magnesium alloys (AZ31, WE43, AZ9) [21], and ceramics such as $\mathrm{Si}_{3} \mathrm{~N}_{4}$ [17] and $\mathrm{Al}_{2} \mathrm{O}_{3}$ [22]. In this study, we attempt to block the toxicity of the SLA printed petri dish by coating the printed parts with parylene $\mathrm{C}$. We assess the toxicity of parylene $\mathrm{C}$ coated petri dish compared with the uncoated groups through zebrafish acute toxicity test.

Zebrafish (Danio rerio) has been shown extensively used in lots of research areas since the finish of a high-quality reference genome sequence assembly in 2013 [23]. It shows an inherent advantage: low maintenance cost, easy availability and high yield of transparent embryos applied in environmental toxicology, disease model, drug screening due to its advantages [24-28]. In this work, we employed zebrafish as proxy models to investigate potential toxicity effects of parylene $C$ coated 3D-printed plastic on aquatic biota. In principle, all test procedure comply with the test guideline has been built as previous [29].

\section{Result}

The acute toxicity test of improved 3D-Printed Parts

Figure $1 \mathrm{~A}$ shows the percentage of survival embryos exposed to uncoated (UT, blue) and parylene $\mathrm{C}$ coated (CT, red) 3D-printed parts compared to that of control embryos (green) through 7 days post fertilization (dpf). While the embryos of UT groups had significantly decreased survival rates $(p<0.05)$, with almost $100 \%$ of the embryos dead by 12 hours post fertilization (hpf) and all dead by $24 \mathrm{hpf}$, the embryos exposed to treated 3D-printed parts had mildly decreased average survival rates compared to those of control embryos.

Similar results were obtained with the hatching rate (Fig. 1B). Because of the fatal effect on embryos, we did not acquire the data of hatching rates for UT 3D-printed parts printers. However, the hatching rate of embryos exposed to the CT group was mildly lower than those of control embryos at $4 \mathrm{dpf}$, with a hatching rate in CT $64.3 \pm 10.6 \%$ vs. $76.0 \pm 3.0 \%$ for the check group $(P=0.14)$ (Fig. 1B). All survived embryos of the CT group and control group hatched at $5 \mathrm{dpf}$.

Teratogenic effect caused by 3D-Printed Parts

We monitor the deformity markers to test the health of embryos throughout the monitoring period of 7 days. Due to the fatal effect of UT 3 D-printed parts printers, the date of this group is missing. In contrast, zebrafish embryos exposed to coated parts had significantly lower rates of malformations. In brief, the percentage of pericardial edema (4 dpf), swim bladder (5 dpf) did not change in CT-exposed embryos 
compared to that of the control embryos. Nevertheless, CT-exposed embryos exhibited a higher heart rate than unexposed control embryos at a statistically significant increase at $4 \mathrm{dpf}(\mathrm{p} \leq 0.05)$ (Fig. 2C).

It is short that the body length $(4 \mathrm{dpf})$ of coated group compare to that of the control $\operatorname{group}(\mathrm{p}<0.001)$ (Fig. 2A).

\section{Materials And Methods}

Producing parylene $\mathrm{C}$ coated STL printed petri dish.

A high-resolution stereolithography printer Lite600 (UnionTech Inc., Guangzhou, Guangdong Province, China) was used to fabricate the petri dishes. Acrylic polymer resin is used as the printing material. The photoinitiator is 2,2-bimethoxy-2-phenylacetophenone. Moderate 3,4-Epoxy cyclohexylmethyl was also added to the raw material. After printing, all constructed parts are removed from the build platform, rinsed, and cleaned with ethanol and acetone. Then the parts are washed with water and dried with air, and the support structures are removed. Finally, all parts are cured in a UV flood chamber for at least five minutes. The petri dish is coated with parylene $C$ with a thickness of $23 \mu \mathrm{m}$ by nano vacuum coating machine L-800 (Guangdong Sante Nano Technology Inc., Dongguan, Guangdong Province, China).

Zebrafish Husbandry and Embryo Collection

Wild type $A B$ strain zebrafish (Danio rerio) obtained from the China Zebrafish Resource Center were used in this study. The Adult zebrafish were maintained were cultured at a light/dark cycle of 14:10 with $28.5 \pm$ $0.5^{\circ} \mathrm{C}$ in a flow-through system $(\mathrm{pH} 7.5 \pm 0.5)$. Males and females were kept separately and fed twice a day with Artemia sp during light cycle. Before spawning, male and female parental zebrafish with a ratio of 1:2 (male to female) were separated in spawning boxes (Esen Corp, Beijing) overnight. The eggs were collected and incubated in egg water during the half an hour after fertilization. Only normally developing embryos were selected for the subsequent experiments.

Phenotype observation on early zebrafish development

Each treatment was exposed to 100 embryos and replicated three times, for a total of 300 embryos used to explore the effectiveness of our improved technique for 3Dprinting methods. The embryos were monitored for their survival, hatching rate, and developmental abnormalities (body length, heart edema, spinal flexure, and absence of swim bladder and heart rate) during 7 days post-fertilization by Nikon SMZ18 microscope (Nikon, Tokyo, Japan).

The numbers of dead, hatching, and developing abnormally (heart edema, absence of swim bladder) individuals were recorded daily during the tests. Embryos without heartbeat and loss of translucency under the microscope were judged to death, removed immediately from the dishes. Survival rate = survival embryos number / total embryos number during $7 \mathrm{~d}$ exposure. The hatching success was identified as opening embryonic membrane and larva swimming up. Hatching rate = hatched embryos number/total embryos number during $7 \mathrm{~d}$ exposure. Pericardial edema was identified as swelling due to an increased 
volume of fluid in the pericardium, which is a portion of the coelomic cavity separating the heart from the body wall. The rates of pericardial edema were calculated as the number of embryos with pericardial edema divided by the number of survival embryos. The HR was manually counted. Percent swim bladder was equal to the number of embryos with swim bladder divided by the total survived number of embryos.

The morphology of the larval was observed and photographed using a Nikon SMZ18 microscope (Nikon, Tokyo, Japan). The images of embryos with pericardial edema were recorded at $3 \mathrm{dpf}$, while embryos with swim bladder at $4 \mathrm{dpf}$.

Measuring the body length of the embryo

The embryos exposed to the CT group at $4 \mathrm{dpf}$ were photographed by Nikon SMZ18 microscope (Nikon, Tokyo, Japan). The body length was quantified using ImageJ software, measuring the distance from the head to the tail of the embryo. About 20 embryos were detected in each group.

Statistical Analysis

Statistical analysis was performed by SPSS 22.0 software (SPSS Inc., Chicago, IL, USA). The significant criteria were set to be $p<0.05$, respectively. Each treatment was replicated three times. The results were reported as the average of three parallel determinations of the mixture of three replicated samples. The figures were completed by GraphPad Prism 8.0 software (GraphPad Software, San Diego, USA). The data were shown as mean \pm standard error.

\section{Discussion}

Our 3D printing ingredient contains acrylate (monomer of photopolymer) and 2,2-bimethoxy-2phenylacetophenone as a photoinitiator. Except for acrylate, we also added moderate 3,4-epoxy cyclohexylmethyl to enhance the mechanical properties of the final product and overcome the drawbacks of a single photopolymer (e.g., low intensity and high anisotropy) [7].

The toxicity of our photopolymer and photoinitiator has been reported in many articles. Unpolymerized acrylate monomer is water-soluble, which means it easily leak into the aqueous medium. In consideration of the water solubility of an acrylate monomer, it may explain why our zebrafish embryos almost dead by $12 \mathrm{hpf}$ and all dead by $24 \mathrm{hpf}(\mathrm{Fig} .1 \mathrm{~A})$, in accord with the recent reports that leaching of compounds to aqueous media from plastic parts is relatively high in the first $24 \mathrm{~h}$ [30]. C.A. Staples et al. test the acute and chronic aquatic toxicity for acrylic acid shows that the acute LC50 (24 h) of Rainbow trout (Oncorhynchus mykiss), Sheepshead minnow (Cyprinodon variegatus), Cladoceran (Daphnia magna), and Mysid shrimp (Mysidopsis bahia) are $45 \mathrm{mg} / \mathrm{l}, 236 \mathrm{mg} / \mathrm{l},>110 \mathrm{mg} / \mathrm{l}$, and $166 \mathrm{mg} / \mathrm{l}$, respectively [31]. The investigation shows that acrylate and urethane acrylate oligomers are toxic or harmful to various species of fish, algae, and water microorganisms.

As for the photoinitiator, though 2,2-bimethoxy-2-phenylacetophenone is poorly soluble in water, it may cause damage to organs through prolonged or repeated exposure, and it is harmful to aquatic life with 
long-lasting effects [32]. Reactive oxygen species (ROS) are the primary metabolites of the photoinitiator, for example, peroxides and peroxy radicals $[33,34]$. Otherwise, incomplete polymerization of photoreactive resins (55- $60 \%$ of polymerization under optimal conditions [35]) can cause more significant levels of toxicity and leaching rate [36]. Hence, our SLA printed parts without coating parylene $C$ are likely to have multiple toxic effects with zebrafish, for the leaching of photopolymer and photoinitiator.

However, in this study, the parylene $\mathrm{C}$ coated group has almost the same level compared to control group, which imply the coating film could block the toxicity of SLA printed parts to zebrafish, despite the hatching rate of embryos exposed to the parylene $\mathrm{C}$ coated group mildly lower than those of control embryos at $4 \mathrm{dpf}$ and parylene $\mathrm{C}$ coated group exhibit a higher heart rate than that of control embryos. The differences between the two groups above demonstrate that slight toxic compounds may exist leaching through the parylene $\mathrm{C}$ film, potentially caused by vestigital after the parylene $\mathrm{C}$ coating step or by molecular exchange for the compounds with lower molecular weight than 300 (e.g., acrylate 86.09, 2,2bimethoxy-2-phenylacetophenone 256.30).

In the past decade, many studies have investigated the toxicity effects of 3D-printed parts. Methods have been exploited to modify the toxicity of 3D-printed parts, including supercritical carbon dioxide [9], sonication of the material in isopropanol [10], and exposure to ultraviolet light [11]. The recent study assessing the toxicity reduced by exposure to ultraviolet light showed a much lower survival and hatch of embryos compared to our method of coating with parylene $C$ [11], indicating that further strategies need to be developed to totally block or reduce the toxicity of SLA printing materials.

In this study, we tested the toxicity of the SLA printed petri dishes whether or not coated with parylene C using early life zebrafish. The UT groups show a violent toxicity effect on the survival and hatch of embryos. On the contrary, the CT group has almost the same level compared to the control group, which implies the coating could significantly reduce the toxicity of STL printed parts to zebrafish. Unfortunately, it does not completely erase the toxicity of these parts. For its potential cardiotoxicity, further strategies for reducing the toxicity are deserved.

This technique can be applied in many aspects. Parylene $\mathrm{C}$ has been proved to be used as an inert bulk coating for metallic biomedical implants to improve their corrosion resistance [18], for it has excellent properties like low water absorption, high dielectric constant, low coefficient of friction, and moderate elongation at break [12]. Although little is known about the durability of the parylene $\mathrm{C}$ coated 3D-printed parts, we can still utilize this cheap and easy technique to test and improve parts that need to be implanted in vivo for the 3D printing is highly customizable. This technique can also contribute to the popularization of SLA printed products, for it can avoid toxicity from daily contact.

Using zebrafish as a model, our research provided a technique to reduce the developmental toxicity of 3Dprinted parts, which currently shows an uplifting promotion to 3D printing usage. However, the wholeorganism health effects of exposure to 3D-printed parts to adult zebrafish are still mysterious. The potential toxicity to whole-organism in animal models and underlying mechanisms need further investigation. 


\section{References}

1. Saroia, J.; Wang, Y.N.; Wei, Q.H.; Lei, M.J.; Li, X.P.; Guo, Y.; Zhang, K. A review on 3D printed matrix polymer composites: its potential and future challenges. Int. J. Adv. Manuf. Technol. 2020, 106, 1695-1721, doi:10.1007/s00170-019-04534-z.

2. Quan, H.Y.; Zhang, T.; Xu, H.; Luo, S.; Nie, J.; Zhu, X.Q. Photo-curing 3D printing technique and its challenges. Bioactive Materials 2020, 5, 110-115, doi:10.1016/j.bioactmat.2019.12.003.

3. Okolie, O.; Stachurek, I.; Kandasubramanian, B.; Njuguna, J. 3D Printing for Hip Implant Applications: A Review. Polymers 2020, 12, doi:10.3390/polym12112682.

4. Rimington, R.P.; Capel, A.J.; Christie, S.D.R.; Lewis, M.P. Biocompatible 3D printed polymers via fused deposition modelling direct C2C12 cellular phenotype in vitro. Lab on a Chip 2017, 17, 2982-2993, doi:10.1039/c7lc00577f.

5. Rimington, R.P.; Capel, A.J.; Player, D.J.; Bibb, R.J.; Christie, S.D.R.; Lewis, M.P. Feasibility and Biocompatibility of 3D-Printed Photopolymerized and Laser Sintered Polymers for Neuronal, Myogenic, and Hepatic Cell Types. Macromol. Biosci. 2018, 18, 12, doi:10.1002/mabi.201800113.

6. Okafor-Muo, O.L.; Hassanin, H.; Kayyali, R.; ElShaer, A. 3D Printing of Solid Oral Dosage Forms: Numerous Challenges With Unique Opportunities. Journal of Pharmaceutical Sciences 2020, 109, 3535-3550, doi:10.1016/j.xphs.2020.08.029.

7. Carve, M.; Wlodkowic, D. 3D-Printed Chips: Compatibility of Additive Manufacturing Photopolymeric Substrata with Biological Applications. Micromachines 2018, 9, doi:10.3390/mi9020091.

8. Walpitagama, M.; Carve, M.; Douek, A.M.; Trestrail, C.; Bai, Y.; Kaslin, J.; Wlodkowic, D. Additives migrating from 3D-printed plastic induce developmental toxicity and neuro-behavioural alterations in early life zebrafish (Danio rerio). Aquat. Toxicol. 2019, 213, 11, doi:10.1016/j.aquatox.2019.105227.

9. Popov, V.K.; Evseev, A.V.; Ivanov, A.L.; Roginski, V.V.; Volozhin, A.I.; Howdle, S.M. Laser stereolithography and supercritical fluid processing or custom-designed implant fabrication. $J$. Mater. Sci.-Mater. Med. 2004, 15, 123-128, doi:10.1023/B:JMSM.0000011812.08185.2a.

10. Ngan, C.G.Y.; O'Connell, C.D.; Blanchard, R.; Boyd-Moss, M.; Williams, R.J.; Bourke, J.; Quigley, A.; McKelvie, P.; Kapsa, R.M.I.; Choong, P.F.M. Optimising the biocompatibility of 3D printed photopolymer constructs in vitro and in vivo. Biomed. Mater. 2019, 14, 12, doi:10.1088/1748605X/ab09c4.

11. Oskui, S.M.; Diamante, G.; Liao, C.; Wei, S.; Grover, W.H.J.E.s.t.I. Assessing and Reducing the Toxicity of 3D-Printed Parts. 2015, 3.

12. Golda-Cepa, M.; Engvall, K.; Hakkarainen, M.; Kotarba, A. Recent progress on parylene $C$ polymer for biomedical applications: A review. Prog. Org. Coat. 2020, 140, 16, doi:10.1016/j.porgcoat.2019.105493.

13. Chang, T.Y.; Yadav, V.G.; De Leo, S.; Mohedas, A.; Rajalingam, B.; Chen, C.L.; Selvarasah, S.; Dokmeci, M.R.; Khademhosseini, A. Cell and protein compatibility of parylene-C surfaces. Langmuir 2007, 23, 11718-11725, doi:10.1021/la7017049. 
14. Tan, C.P.; Craighead, H.G. Surface Engineering and Patterning Using Parylene for Biological Applications. Materials 2010, 3, 1803-1832, doi:10.3390/ma3031803.

15. Kuo, J.T.W.; Kim, B.J.; Hara, S.A.; Lee, C.D.; Gutierrez, C.A.; Hoang, T.Q.; Meng, E. Novel flexible Parylene neural probe with 3D sheath structure for enhancing tissue integration. Lab on a Chip 2013, 13, 554-561, doi:10.1039/c2lc40935f.

16. Temiz, Y.; Ferretti, A.; Leblebici, Y.; Guiducci, C. A comparative study on fabrication techniques for onchip microelectrodes. Lab on a Chip 2012, 12, 4920-4928, doi:10.1039/c2lc40582b.

17. Hassler, C.; von Metzen, R.P.; Ruther, P.; Stieglitz, T. Characterization of parylene $C$ as an encapsulation material for implanted neural prostheses. Journal of biomedical materials research. Part B, Applied biomaterials 2010, 93, 266-274, doi:10.1002/jbm.b.31584.

18. Cieslik, M.; Engvall, K.; Pan, J.; Kotarba, A. Silane-parylene coating for improving corrosion resistance of stainless steel 316 implant material. Corrosion Science 2011, 53, 296-301, doi:10.1016/j.corsci.2010.09.034.

19. Youssefian, S.; Rahbar, N. Nano-scale adhesion in multilayered drug eluting stents. Journal of the Mechanical Behavior of Biomedical Materials 2013, 18, 1-11, doi:10.1016/j.jmbbm.2012.11.001.

20. Bar-Cohen, Y.; Silka, M.J.; Hill, A.C.; Pruetz, J.D.; Chmait, R.H.; Zhou, L.; Rabin, S.M.; Norekyan, V.; Loeb, G.E. Minimally Invasive Implantation of a Micropacemaker Into the Pericardial Space. CirculationArrhythmia and Electrophysiology 2018, 11, doi:10.1161/circep.118.006307.

21. Surmeneva, M.A.; Vladescu, A.; Cotrut, C.M.; Tyurin, A.I.; Pirozhkova, T.S.; Shuvarin, I.A.; Elkin, B.; Oehr, C.; Surmenev, R.A. Effect of parylene $C$ coating on the antibiocorrosive and mechanical properties of different magnesium alloys. Applied Surface Science 2018, 427, 617-627, doi:10.1016/j.apsusc.2017.08.066.

22. Xie, X.Z.; Rieth, L.; Williams, L.; Negi, S.; Bhandari, R.; Caldwell, R.; Sharma, R.; Tathireddy, P.; Solzbacher, F. Long-term reliability of Al2O3 and Parylene $\mathrm{C}$ bilayer encapsulated Utah electrode array based neural interfaces for chronic implantation. J. Neural Eng. 2014, 11, 9, doi:10.1088/17412560/11/2/026016.

23. Howe, K.; Clark, M.D.; Torroja, C.F.; Torrance, J.; Berthelot, C.; Muffato, M.; Collins, J.E.; Humphray, S.; McLaren, K.; Matthews, L.; et al. The zebrafish reference genome sequence and its relationship to the human genome (vol 496, pg 498, 2013). Nature 2014, 505, 248-248, doi:10.1038/nature12813.

24. McGrath, P.; Li, C.Q. Zebrafish: a predictive model for assessing drug-induced toxicity. Drug Discov. Today 2008, 13, 394-401, doi:10.1016/j.drudis.2008.03.002.

25. Scholz, S.; Fischer, S.; Gundel, U.; Kuster, E.; Luckenbach, T.; Voelker, D. The zebrafish embryo model in environmental risk assessment - applications beyond acute toxicity testing. Environ. Sci. Pollut. Res. 2008, 15, 394-404, doi:10.1007/s11356-008-0018-z.

26. Kalueff, A.V.; Stewart, A.M.; Gerlai, R. Zebrafish as an emerging model for studying complex brain disorders. Trends Pharmacol. Sci. 2014, 35, 63-75, doi:10.1016/j.tips.2013.12.002.

27. Zon, L.I.; Peterson, R.T. In vivo drug discovery in the zebrafish. Nat. Rev. Drug Discov. 2005, 4, 35-44, doi:10.1038/nrd1606. 
28. Mu, X.Y.; Shen, G.M.; Huang, Y.; Luo, J.B.; Zhu, L.Z.; Qi, S.Z.; Li, Y.R.; Wang, C.J.; Li, X.F. The enantioselective toxicity and oxidative stress of beta-cypermethrin on zebrafish. Environ. Pollut. 2017, 229, 312-320, doi:10.1016/j.envpol.2017.05.088.

29. OECD,OECD Guidelines for the Testing of Chemicals, Fish Embryo Acute Toxicity (FET) Test OECD, Paris, France (2013)No. 236.

30. Biocompatibility of Dental Materials.

31. Staples, C.A.; Murphy, S.R.; McLaughlin, J.E.; Leung, H.W.; Cascieri, T.C.; Farr, C.H. Determination of selected fate and aquatic toxicity characteristics of acrylic acid and a series of acrylic esters. Chemosphere 2000, 40, 29-38, doi:10.1016/s0045-6535(99)00228-3.

32. https://echa.europa.eu/information-on-chemicals/cl-inventory-database/-/discli/details/105868.

33. Lago, M.A.; de Quiros, A.R.B.; Sendon, R.; Bustos, J.; Nieto, M.T.; Paseiro, P. Photoinitiators: a food safety review. Food Addit. Contam. Part A-Chem. 2015, 32, 779-798, doi:10.1080/19440049.2015.1014866.

34. Sangermano, M. Advances in cationic photopolymerization. Pure Appl. Chem. 2012, 84, 2089-2101, doi:10.1351/pac-con-12-04-11.

35. Ferracane, J.L.; Condon, J.R. POSTCURE HEAT-TREATMENTS FOR COMPOSITES - PROPERTIES AND FRACTOGRAPHY. Dent. Mater. 1992, 8, 290-295, doi:10.1016/0109-5641(92)90102-i.

36. Melchels, F.P.W.; Feijen, J.; Grijpma, D.W. A review on stereolithography and its applications in biomedical engineering. Biomaterials 2010, 31, 6121-6130, doi:10.1016/j.biomaterials.2010.04.050.

\section{Declarations}

\section{Competing interests.}

The authors declare that they have no competing interests.

The overall study and animal experiments conformed to the guidelines and regulatory standards of the Institutional Animal Care and Use Committee of China Agricultural University and Ethics Committee of Henan Children's Hospital.

\section{Figures}


A

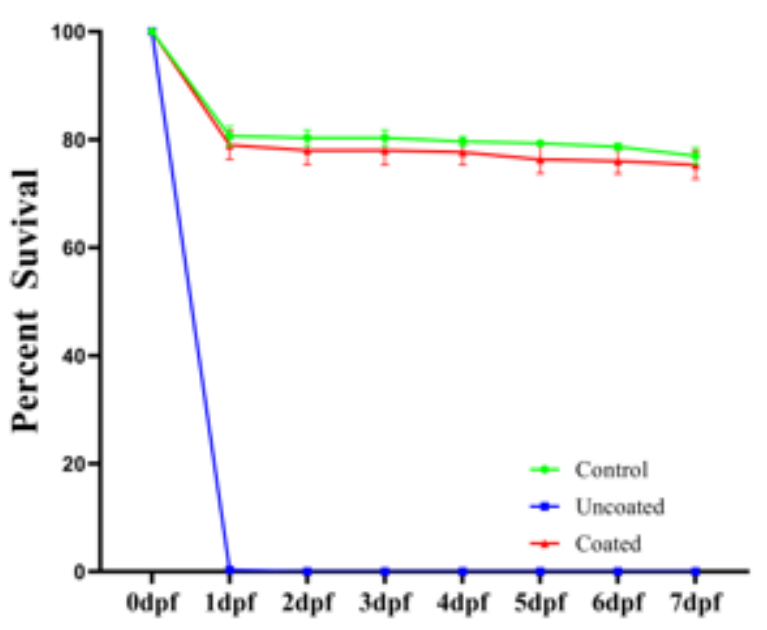

B

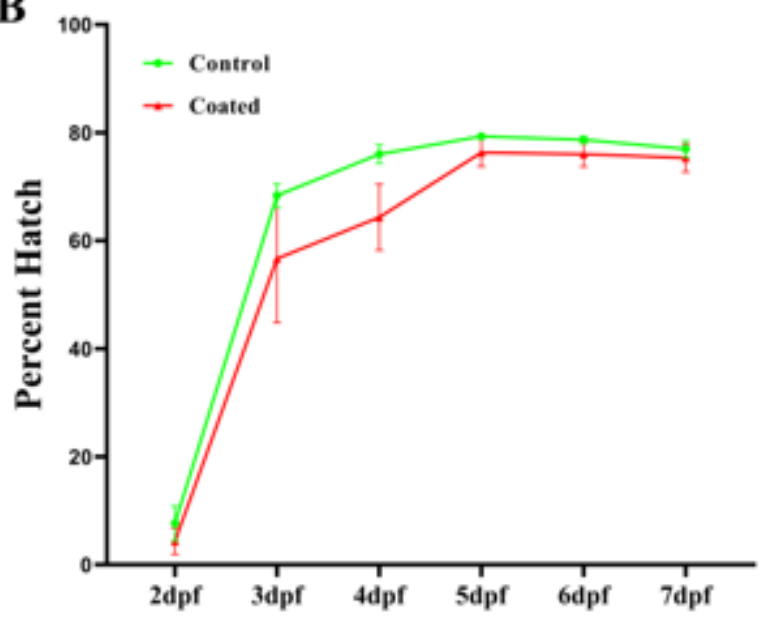

\section{Figure 1}

The survival and hatch analysis of zebrafish embryo treated after 3D-print parts. (A) Survival rates of zebrafish embryos exposed to 3D-printed parts from CT (red green), UT (blue) and control embryos that were not exposed to printed parts (green). Each exposure represents three replicates with 100 embryos in each replicate. Embryos exposed to UT parts had significantly lower survival rates (almost $100 \%$ dead) by $12 \mathrm{hpf}$ when compared to those of control embryos $(p<0.001)$, with no STL-exposed embryo surviving past day 7. Inversely, embryos exposed to parylene $C$ coated parts did not have significantly decreased survival rates compared to those of control embryos $(p>0.05)$. (B) Hatching rates for the CT exposed and control. We did not acquire the data of hatching rates for UT 3 D-printed parts due to its fatal effect on embryos. On the contrary, embryos exposed to CT parts did not have significantly lower hatching rates in the embryos $(p>0.05)$. These results show that coated treated STL-printed parts had almost no toxicity on the survival and hatch rate of embryos exposed to the CT parts fare almost as well as control embryos groups. 


\section{A}
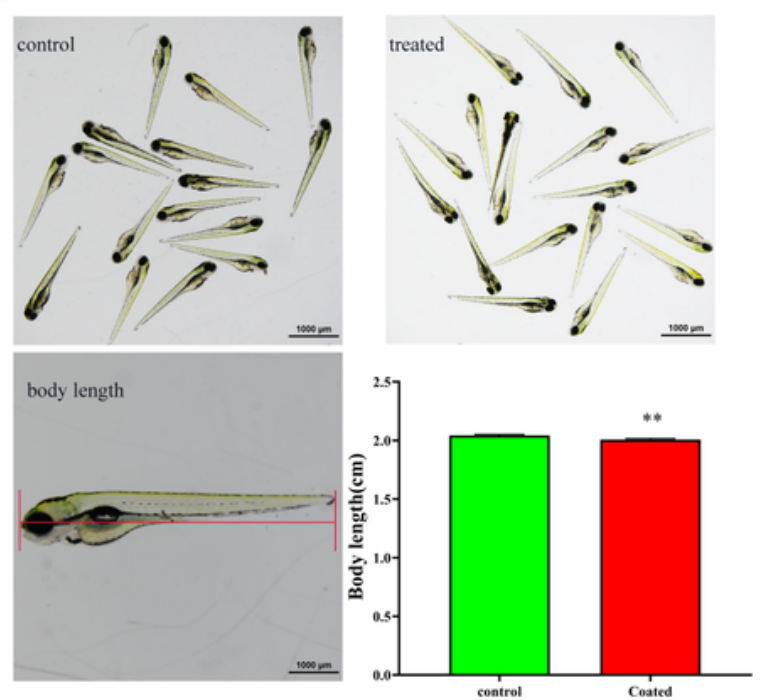

B
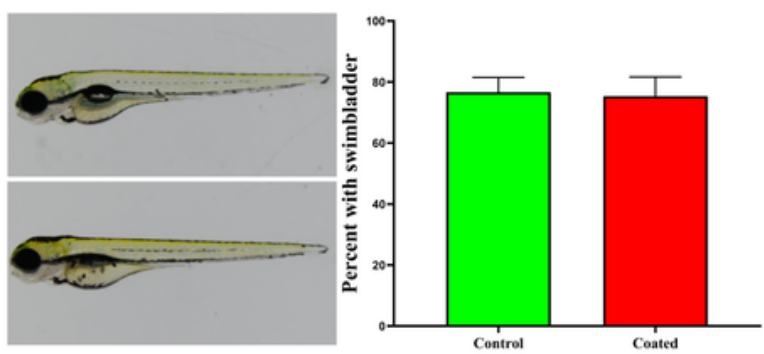

C
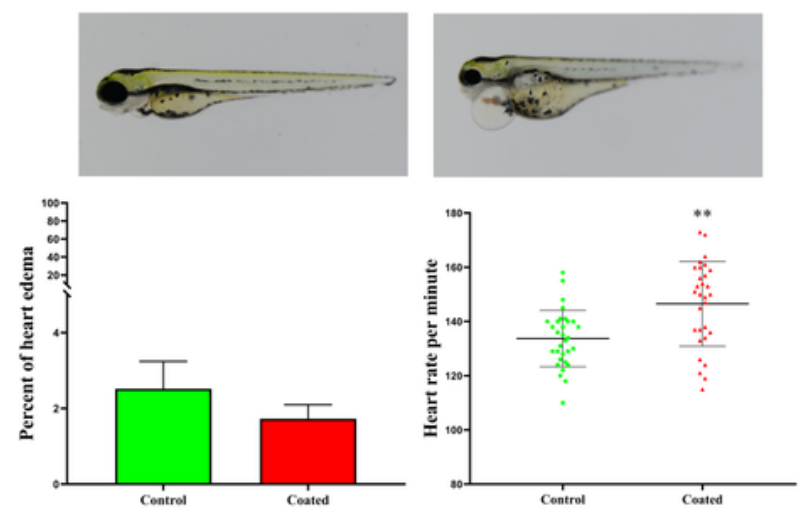

\section{Figure 2}

Effect of exposure to $3 \mathrm{D}$-printed parts printers on zebrafish malformation rate. (A) Embryos exposed to coated 3D-printed parts printers exhibited a reduced body length compared with that of controls at $4 \mathrm{dpf}$ (parylene $\mathrm{C}$ coated group $2.0058 \pm 0.0708$, control group $2.0419 \pm 0.0570, p=0.0067$ ). Body length was measured along the body axis using image $\mathrm{J}$ software. Statistically significant differences are indicated by asterisks ( $p<0.05$, Student's t-test). (B) Parylene $C$ coated parts did not have any effect on the 
development of swim bladders compared with the uncoated group. (C) Pericardial edema percent in the parylene $\mathrm{C}$ coated group shows no difference compared with that of controls at $4 \mathrm{dpf}$. In contrast, zebrafish embryos parylene $\mathrm{C}$ coated parts caused an increase of the HR at $5 \mathrm{dpf}$ compared to control embryos (parylene $C$ coated group $146 \pm 16$ bpm, control group $135 \pm 11 \mathrm{bpm}, \mathrm{p}=0.0034$ ). The scale bar represents $1000 \mu \mathrm{m}$. Values are presented as mean \pm S.E. 